PROCEEDINGS OF THE

AMERICAN MATHEMATICAL SOCIETY

Volume 126, Number 6, June 1998, Pages 1827-1833

S 0002-9939(98)04426-8

\title{
THERE IS A PARACOMPACT Q-SET SPACE IN ZFC
}

\author{
ZOLTAN T. BALOGH \\ (Communicated by Franklin D. Tall)
}

\begin{abstract}
We construct a paracompact space $Q X$ such that every subset of $Q X$ is an $F_{\sigma}$-set, yet $Q X$ is not $\sigma$-discrete. We will construct our space not to have a $G_{\delta}$-diagonal, which answers questions of A.V. Arhangel'skiǐ and D. Shakhmatov on cleavable spaces.
\end{abstract}

\section{INTRODUCTION}

In this paper we will construct a hereditarily paracompact, perfectly normal Qset space $Q X$ without a quasi- $G_{\delta}$-diagonal. $Q X$ answers questions on $\mathrm{Q}$-set spaces, and on cleavable spaces of A.V. Arhangel'skiǐ.

A topological space $X$ is a $\mathbf{Q}$-set space [B] if every subset of $X$ is a $G_{\delta}$-set and $X$ is not $\sigma$-discrete. H. Junnila [J] (and Bregman-Shapirovskií-Soštak) asked whether there were any Q-set spaces in ZFC. This problem was answered affirmatively for regular Q-set spaces, and the question was raised whether there are (perfectly) normal Q-set spaces [B]. In this paper we shall combine the technique of the regular examples with a new inductive method to show not only that the answer is yes, but that one can also construct paracompact examples.

A.V. Arhangel'skiĩ and D.B. Shakhmatov [AS], [A1] raised the question whether every cleavable space has a $G_{\delta}$-diagonal. Arhangel'skiǐ [A2] also asked whether spaces cleavable over the rationals had to be $\sigma$-discrete or had to possess $G_{\delta^{-}}$ diagonals. Since normal Q-set spaces are cleavable and also cleavable over the rationals [A2], and our space $Q X$ will be constructed not to have a $G_{\delta}$-diagonal, it settles all of the above questions in the negative. (It should be pointed out here, that a $\mathrm{Q}$-space is defined in [A2] to be a space whose every subset is an $F_{\sigma}$-set. Thus, Q-set spaces are precisely the non- $\sigma$-discrete Q-spaces).

$Q X$ will have cardinality $c^{+}$, which is necesssary only to make it not have a $G_{\delta}$-diagonal. If we only want to construct a paracompact Q-set space, then it can be done on $c$ (Theorem 2.1).

Terminology and notation. We use the standard terminology and notation of set-theoretic topology (see $[\mathrm{KV}]$ ). $\pi$ will always denote first projection, i.e. $\pi A=\{a$ : there is a $b$ with $\langle a, b\rangle \in A\}$. A sequence of $\left\langle\mathcal{G}_{m}\right\rangle_{m \in \omega}$ of families of open subsets of a space $X$ is said to be a quasi- $G_{\delta}$-diagonal, if for every $x \in$ $X, \bigcap\left\{s t\left(x, \mathcal{G}_{m}\right): m \in \omega\right.$ and $\left.x \in \bigcup \mathcal{G}_{m}\right\}=\{x\}$.

Received by the editors August 24, 1995.

1991 Mathematics Subject Classification. Primary 54Dxx.

Key words and phrases. Paracompact, Q-set space, $G_{\delta}$-diagonal, cleavable.

Research supported by NSF Grant DMS-9108476. 


\section{The SpaCe $Q X$}

Theorem 1.1. There is a (hereditarily) paracompact, perfectly normal Q-set space $Q X$ without a quasi- $G_{\delta}$-diagonal.

Proof. The underlying set of $Q X$ is $c^{+}$, the first cardinal bigger than the continum $c$. The topology of $Q X$ will be inductively defined in $\lambda=2^{c^{+}}$steps. For the purposes of making every subset of $Q X$ a $G_{\delta^{-}}$-set, let $\left\langle Y_{\xi}\right\rangle_{\xi<\lambda}$ be a one-to-one listing of all subsets of $c^{+}$. Also, let $\left\langle U_{\xi}\right\rangle_{\xi<\lambda}$ be a list of all subsets of $c^{+} \times c^{+}$such that $U_{0}=\phi$ and each subset is listed $\lambda$ times. This second list will, in particular, mention codes for all future open covers of $Q X$. If such an open cover first occurs at step $\xi$, then we'll add a clopen partition refining that cover to the topology of $Q X$. To carry out the program above we shall define, by induction of $\xi<\lambda$,

(a) a function $g_{\xi}: c^{+} \longrightarrow \omega+1$;

(b) a number $h(\xi) \in\{0,1\}$;

(c) a function $w_{\xi}: c^{+} \longrightarrow c^{+} \backslash \omega$ if $h(\xi)=1$.

We will set

(a') $G_{\xi n}=\left\{\alpha<c^{+}: g_{\xi}(\alpha) \geq n\right\}$ for every $n \in \omega$;

(b') $H=\{\xi<\lambda: h(\xi)=1\}$;

(c') $W_{\xi \rho}=\left\{\alpha<c^{+}: w_{\xi}(\alpha)=\rho\right\}$ for every $\xi \in H$ and $\rho$ with $\omega \leq \rho<c^{+}$.

A subbase for the topology $\tau_{Q}$ of $Q X$ will be

$$
\mathcal{B}=\left\{G_{\xi n}: \xi<\lambda, n \in \omega\right\} \cup\left\{W_{\xi \rho}: \xi \in H \text { and } \rho \in c^{+} \backslash \omega\right\} .
$$

Adding the $G_{\xi n}$ 's will make every subset of $X$ a $G_{\delta^{-}}$-set. $\left\{W_{\xi \rho}: \rho \in c^{+} \backslash \omega\right\}$ will be a clopen partition refinement of the open cover coded by rows $\omega \leq \rho<c^{+}$of $U_{\xi} \subset c^{+} \times c^{+}$if $\xi \in H$.

In order to make sure that $Q X$ does not have a quasi- $G_{\delta^{-}}$-diagonal we will need the concept of a control pair. We will say that $\langle A, \underline{u}\rangle$ is a control pair if

(C-1) $A \in\left[c^{+}\right]^{\omega}$;

(C-2) $\underline{u}=\left\langle u_{0}, u_{1}, u_{2}\right\rangle$, and $u_{0}, u_{1}, u_{2}$ are functions with domain $A$;

(C-3) for every $\alpha \in A, u_{0}(\alpha) \in[P(A) \times \omega]^{<\omega}, u_{1}(\alpha) \in[P(A \times A)]^{<\omega}$ and $u_{2}(\alpha) \in$ $\left[P(A \times A) \backslash u_{1}(\alpha)\right]^{<\omega}$

(C-4) if $\alpha, \alpha^{\prime} \in A$ and $\alpha \neq \alpha^{\prime}$, then $\pi u_{0}(\alpha) \cap \pi u_{0}\left(\alpha^{\prime}\right)=\phi$ and $u_{1}(\alpha) \cap u_{1}\left(\alpha^{\prime}\right)=\phi$.

(Note that $\pi u_{0}(\alpha)=\left\{B \subset A\right.$ there is an $n \in \omega$ with $\left.\langle B, n\rangle \in u_{0}(\alpha)\right\}$ ).

Roughly speaking, $\langle A, u\rangle$ will code a countable approximation to a neighborhood assignment in $Q X$. Let $\left\langle A_{\beta}, \underline{u}_{\beta}\right\rangle_{\beta<c^{+}}$list all control pairs, mentioning each $c^{+}$ times.

The last ingredient we need is the notion of an initially $\xi$-open set. A subset $E \subset c^{+}$will be called initially $\xi$-open, if $E$ is an open subset in the topology generated by

$$
\mathcal{B}_{\xi}=\{X\} \cup\left\{G_{\eta n}: \eta<\xi, n \in \omega\right\} \cup\left\{W_{\eta \rho}: \eta<\xi, h(\eta)=1 \text { and } \rho \in c^{+} \backslash \omega\right\} .
$$

For every $\xi<\lambda$ and $\rho \in c^{+} \backslash \omega$, let $U_{\xi \rho}=\left\{\gamma<c^{+}:\langle\gamma, \rho\rangle \in U_{\xi}\right\}$.

We are going to construct $g_{\xi}, h(\xi)$ and $w_{\xi}($ if $h(\xi)=1$ ) in such a way that the following hypotheses are satisfied:

$\left(1_{\xi}\right)$ for every $\beta<c^{+}, g_{\xi}(\beta)=\omega$ iff $\beta \in Y_{\xi}$;

$\left(2_{\xi}\right)$ if $\alpha<\beta<c^{+}$and $\left\langle Y_{\xi} \cap A_{\beta}, n\right\rangle \in u_{0 \beta}(\alpha)$, then $g_{\xi}(\beta) \geq n$; 
$\left(3_{\xi}\right) h(\xi)=1$ if and only if $\left\langle U_{\xi \rho}\right\rangle_{\rho \in c^{+} \backslash \omega}$ is a cover of $c^{+}$consisting of initially $\xi$-open sets and there is no $\xi^{\prime}<\xi$ such that $U_{\xi^{\prime}}=U_{\xi}$ and $\left\langle U_{\xi^{\prime} \rho}\right\rangle_{\rho \in c^{+} \backslash \omega}$ is a cover by initially $\xi^{\prime}$-open sets;

$\left(4_{\xi}\right)$ if $h(\xi)=1$, then

(a) for every $\beta<c^{+}, \beta \in U_{\xi w_{\xi}(\beta)}$;

(b) if $\alpha<\beta<c^{+}, U_{\xi} \cap\left(A_{\beta} \times A_{\beta}\right) \in u_{1 \beta}(\alpha)$ and $\beta \in U_{\xi w_{\xi}(\alpha)}$, then $w_{\xi}(\beta)=w_{\xi}(\alpha)$;

(c) if $\alpha<\beta<c^{+}, U_{\xi} \cap\left(A_{\beta} \times A_{\beta}\right) \in u_{2 \beta}(\alpha)$ and $\beta \in U_{\xi w_{\xi}(\alpha)}$, then there is an $\alpha^{\prime} \in A_{\beta}$ such that $w_{\xi}(\beta)=w_{\xi}\left(\alpha^{\prime}\right)$.

Let us now pass to the construction. Suppose that $\xi<\lambda$ and that we are done for $\eta<\xi$.

We are going to define $g_{\xi}(\beta) \in \omega+1$ by induction on $\beta<c^{+}$. Suppose we are done for every $\alpha<\beta$. We split the definition into two cases.

Case 1. Suppose that there is no $\alpha<\beta$ and $n \in \omega$ with $\left\langle Y_{\xi} \cap A_{\beta}, n\right\rangle \in u_{0 \beta}(\alpha)$. Then let $g_{\xi}(\beta)=\omega$ if $\beta \in Y_{\xi}$, and $g_{\xi}(\beta)=0$ if $\beta \notin Y_{\xi}$.

Case 2. Suppose now that there is an $\alpha<\beta$ such that $\left\langle Y_{\xi} \cap A_{\beta}, n\right\rangle \in u_{0 \beta}(\alpha)$ for some $n \in \omega$. By (C-4), there is only one such $\alpha$; furthermore, since $u_{0 \beta}(\alpha)$ is a finite set, there are only finitely many such $n \in \omega$. Set $g_{\xi}(\beta)=\max \{n \in \omega$ : $\left.\left\langle Y_{\xi} \cap A_{\beta}, n\right\rangle \in u_{0 \beta}(\alpha)\right\}$, if $\beta \notin Y_{\xi}$, and $g_{\xi}(\beta)=\omega$, if $\beta \in Y_{\xi}$.

With these definitions, $\left(1_{\xi}\right)$ and $\left(2_{\xi}\right)$ are clearly satisfied.

Note that $\left(3_{\xi}\right)$ permits exactly one of 0 or 1 to be $h(\xi)$ and define $h(\xi)$ according to $(3 \xi)$.

If $h(\xi)=0$, then leave $w_{\xi}$ undefined.

Suppose now that $h(\xi)=1$. We are going to define $w_{\xi}(\beta)$ by induction on $\beta<c^{+}$. Suppose that we are done for every $\alpha<\beta$. We consider three cases.

Case 1. Suppose that there is an $\alpha<\beta$ such that $U_{\xi} \cap\left(A_{\beta} \times A_{\beta}\right) \in u_{1 \beta}(\alpha)$ and $\beta \in U_{\xi w_{\xi}(\alpha)}$. Note that by (C-4) there is only one such $\alpha$ and that $\alpha \in A_{\beta}$. Set $w_{\xi}(\beta)=w_{\xi}(\alpha)$.

Case 2. Suppose that Case 1 does not hold, but there is an $\alpha<\beta$ such that $U_{\xi} \cap\left(A_{\beta} \times A_{\beta}\right) \in u_{2 \beta}(\alpha)$ and $\beta \in U_{\xi w_{\xi}(\alpha)}$. Note that every such $\alpha$ belongs to $A_{\beta}$. Fix one such $\alpha$ and set $w_{\xi}(\beta)=w_{\xi}(\alpha)$ for that $\alpha$.

Case 3. Suppose that neither Case 1 nor Case 2 holds. Then pick any $\rho \in c^{+} \backslash \omega$ with $\beta \in U_{\xi \rho}$ (since $\left\langle U_{\xi \rho}\right\rangle_{\rho \in c^{+} \backslash \omega}$ is a cover of $c^{+}$, there is at least one such $\rho$ ) and set $w_{\xi}(\beta)=\rho$.

It is easy to check that $\left(4_{\xi}\right)$ is satisfied in all of the cases above.

To finish our construction, let $\tau$ denote the topology generated by

$$
\mathcal{B}=\bigcup_{\xi<\lambda} \mathcal{B}_{\xi}=\left\{G_{\xi n}: \xi<\lambda, n \in \omega\right\} \cup\left\{W_{\xi \rho}: \rho \in c^{+} \backslash \omega, \xi<\lambda \text { and } h(\xi)=1\right\}
$$

as a subbase.

Let $Q X=\left\langle c^{+}, \tau\right\rangle$. The rest of the proof consists of checking that this space possesses the desired properties.

I. To check that every subset of $Q X$ is a $G_{\delta}$-set, let $Y \subset c^{+}$. Then there is a $\xi<\lambda$ such that $Y=Y_{\xi}$. By $\left(1_{\xi}\right), Y=Y_{\xi}=\bigcap_{n \in \omega} G_{\xi n}$, i.e. $Y$ is a $G_{\delta}$-set.

Note that since complements of singletons are $G_{\delta}$-sets (and thus, open sets), every singleton set is closed, i.e. $X$ is a $T_{1}$-space. 
II. In order to show that $Q X$ is ultraparacompact it is enough to prove that every open cover of $Q X$ has a refinement which is a partition of $c^{+}$into pairwise disjoint clopen sets. So let $\mathcal{U}$ be an arbitrary open cover of $Q X$ and let $\left\langle U_{\rho}\right\rangle_{\rho \in c^{+} \backslash \omega}$ be a list of $\mathcal{U} \cup\{\phi\}$. Let $U=\bigcup_{\rho \in c^{+} \backslash \omega} U_{\rho} \times\{\rho\}$. Since on the list $\left\langle U_{\xi}\right\rangle_{\xi<\lambda}$ of all subsets of $c^{+} \times c^{+}, U$ is listed $\lambda$ times, and because $c f(\lambda)=c f\left(2^{c^{+}}\right)>c^{+}$, there is a first $\xi<\lambda$ such that $U_{\xi}=U$ and $U_{\rho}$ is initially $\xi$-open for every $\rho \in c^{+} \backslash \omega$. For this $\xi,\left\langle U_{\xi \rho}\right\rangle_{\rho \in c^{+} \backslash \omega}=\left\langle U_{\rho}\right\rangle_{\rho \in c^{+} \backslash \omega}$ and $h(\xi)=1$. Therefore $w_{\xi}: c^{+} \rightarrow c^{+} \backslash \omega$ is defined and $\left\langle W_{\xi \rho}\right\rangle_{\rho \in c^{+} \backslash \omega}$ is a refinement of $\left\langle U_{\rho}\right\rangle_{\rho \in c^{+} \backslash \omega}$ to a partition of $Q X$ into clopen subsets.

III. Perfect normality of $X$ follows from I and II.

IV. The rest of the proof consists of showing that $Q X$ does not have a quasi$G_{\delta}$-diagonal. From this it automatically follows that $Q X$ is not $\sigma$-discrete, because a $\sigma$-discrete space in which every point is a $G_{\delta^{-}}$set has a quasi- $G_{\delta^{-}}$-diagonal.

First, for every $\langle\xi, \cdot\rangle \in \lambda \times \omega \cup H \times\left(c^{+} \backslash \omega\right)$, let $Q_{\xi}=G_{\xi n}$ if $\langle\xi, \cdot\rangle=\langle\xi, n\rangle$ for some $n \in \omega$, and let $Q_{\xi}$. $=W_{\xi \rho}$ if $\langle\xi \cdot\rangle=\langle\xi, \rho\rangle$ for some $\rho \in c^{+} \backslash \omega$. Further, let us note that $\left\langle U_{\xi}\right\rangle_{\xi \in H}$ is a one-to-one list.

Now, let us consider an arbitrary sequence $\left\langle\mathcal{G}_{m}\right\rangle_{m \in \omega}$ of families of open subsets of $Q X$. We are going to show that $\left\langle\mathcal{G}_{m}\right\rangle_{m \in \omega}$ does not form a quasi- $G_{\delta}$-diagonal in $Q X$. For this purpose we can assume without loss of generality that each $\mathcal{G}_{m}$ is a non-empty family of non-empty sets and that $\mathcal{G}=\bigcup_{m \in \omega} \mathcal{G}_{m}$ covers $Q X$.

For each $m \in \omega$ let $q_{m}: \bigcup \mathcal{G}_{m} \rightarrow\left[\lambda \times \omega \cup H \times\left(c^{+} \backslash \rho\right)\right]^{<\omega}$ code a refinement of $\mathcal{G}_{m}$ by basic open sets, i.e. for every $\alpha \in \bigcup \mathcal{G}_{m}$ there is a $G \in \mathcal{G}_{m}$ such that

$$
\alpha \in Q_{m}(\alpha)=\bigcap\left\{Q_{\xi}:\langle\xi, \cdot\rangle \in q_{m}(\alpha)\right\} \subset G .
$$

By extending $q_{m}(\alpha)$, if necessary, we can assume without loss of generality that for every $m \in \omega$ and $\alpha \in \bigcup \mathcal{G}_{m}$,

(F) if $\langle\xi, \rho\rangle \in q_{m}(\alpha) \cap\left(H \times\left(c^{+} \backslash \omega\right)\right)$, then there is a $t_{\xi m}(\alpha) \subset q_{m}(\alpha)$ such that (F-a) $\langle\eta, \cdot\rangle \in t_{\xi m}(\alpha)$ implies $\eta<\xi$;

(F-b) $\alpha \in T_{\xi m}(\alpha)=\bigcap\left\{Q_{\eta}:\langle\eta, \cdot\rangle \in t_{\xi m}(\alpha)\right\} \subset U_{\xi \rho}$.

This can be done because if $\langle\xi, \rho\rangle \in q_{m}(\alpha) \cap\left(H \times\left(c^{+} \backslash \omega\right)\right)$, then $U_{\xi \rho}$ is an initially $\xi$-open set containing $\alpha$. Also, note that $t_{\xi m}(\alpha)$ does not depend on $\rho$, because $\xi$ and $\alpha$ determine $\rho$ through the condition $\alpha \in W_{\xi \rho}$.

In order to prove that $\left\langle\mathcal{G}_{m}\right\rangle_{m \in \omega}$ is not a quasi- $G_{\delta}$-diagonal it is enough to find two distinct elements $\beta_{0}, \beta_{1}$ of $c^{+}$such that $\left\{m \in \omega: \beta_{0} \in \bigcup \mathcal{G}_{m}\right\}=\left\{m \in \omega: \beta_{1} \in\right.$ $\left.\cup \mathcal{G}_{m}\right\}$, and if we denote this subset of $\omega$ by $\mathrm{N}$, then

(*) for every $m \in N$ there is an $\alpha \in \bigcup \mathcal{G}_{m}$ such that $\left\{\beta_{0}, \beta_{1}\right\} \subset Q_{m}(\alpha)$.

In order to find such $\beta_{0}$ and $\beta_{1}$, let $M$ be a countable elementary submodel of $H\left(\left(2^{c^{+}}\right)^{+}\right)$such that $\left\langle Y_{\xi}\right\rangle_{\xi<\lambda},\left\langle U_{\xi}\right\rangle_{\xi \in H},\left\langle g_{\xi}\right\rangle_{\xi<\lambda},\left\langle w_{\xi}\right\rangle_{\xi \in H},\left\langle\mathcal{G}_{m}\right\rangle_{m \in \omega}$ and $\left\langle q_{m}\right\rangle_{m \in \omega}$ are all elements of $M$. Let $A^{*}=M \cap c^{+}$and let $\left\langle A^{*}, \underline{u}^{*}\right\rangle$ be a control pair such that if $v: c^{+} \rightarrow\left[\lambda \times \omega \cup H \times\left(c^{+} \backslash \omega\right)\right]^{<\omega}$ is an infinite partial function in $M$ and $\{\pi v(\alpha): \alpha \in \operatorname{dom}(v)\}$ forms a $\Delta$-system with root $r_{v}$, then there is an $\alpha \in A^{*}$ such that

$$
\begin{aligned}
& u_{0}^{*}(\alpha)=\left\{\left\langle Y_{\xi} \cap A^{*}, n\right\rangle:\langle\xi, n\rangle \in v(\alpha) \text { and } \xi \notin r_{v}\right\} ; \\
& u_{1}^{*}(\alpha)=\left\{U_{\xi} \cap\left(A^{*} \times A^{*}\right): \xi \in\left(\pi v(\alpha) \backslash r_{v}\right) \cap H\right\} ; \\
& u_{2}^{*}(\alpha)=\left\{U_{\xi} \cap\left(A^{*} \times A^{*}\right): \xi \in r_{v} \cap H\right\} .
\end{aligned}
$$


To see that such a control pair $\left\langle A^{*}, u^{*}\right\rangle$ exists, let $\left\langle v_{k}\right\rangle_{k \in \omega}$ list all functions above.

By induction on $k \in \omega$, define a sequence $\left\langle\alpha_{k}\right\rangle_{k \in \omega}$ of distinct elements of $A^{*}=$ $c^{+} \cap M$ in such a way that $\pi v_{k}\left(\alpha_{k}\right)-r_{v_{k}}(k \in \omega)$ are pairwise disjoint. Then define $u_{j}^{*}\left(\alpha_{k}\right)(j=0,1,2)$ as in (D), writing $\alpha_{k}$ and $v_{k}$ in place of $\alpha$ and $v$, and set $u_{j}^{*}(\alpha)=$ $\emptyset$ for $\alpha \in A^{*} \backslash\left\{\alpha_{k}: k \in \omega\right\}$. Then $\left\langle A^{*}, \underline{u}^{*}\right\rangle$, where $\underline{u}^{*}(\alpha)=\left\langle u_{0}^{*}(\alpha), u_{1}^{*}(\alpha), u_{2}^{*}(\alpha)\right\rangle$, is a control pair as desired. (Properties (C-3) and (c-4) of a control pair follow from (D), because $M$ is an elementary submodel and the lists $\left\langle Y_{\xi}\right\rangle_{\xi<\lambda},\left\langle U_{\xi}\right\rangle_{\xi \in H}$ are one-to-one.)

Now, let $\beta_{0}, \beta_{1}>\sup A^{*}$ be such that

(i) $\left\langle A^{*}, \underline{u}^{*}\right\rangle=\left\langle A_{\beta i}, \underline{u}_{\beta i}\right\rangle$ for $\mathrm{i}=0,1$;

(ii) $\left\{m \in \omega: \beta_{0} \in \bigcup \mathcal{G}_{m}\right\}=\left\{m \in \omega: \beta_{1} \in \bigcup \mathcal{G}_{m}\right\}=N$;

(iii) for every $m \in N$

(iii-a) $q_{m}\left(\beta_{0}\right) \cap M=q_{m}\left(\beta_{1}\right) \cap M$ (denote this set by $\left.y_{m}\right)$;

(iii-b) $\left\{\xi \in M \cap H:\left\langle\xi, w_{\xi}\left(\beta_{0}\right)\right\rangle \in q_{m}\left(\beta_{0}\right)\right\}=\left\{\xi \in M \cap H:\left\langle\xi, w_{\xi}\left(\beta_{1}\right)\right\rangle \in q_{m}\left(\beta_{1}\right)\right\}$ (denote this set by $t_{m}$ ). Note that (iii-a) and (iii-b) together imply

(iii-c) $\pi q_{m}\left(\beta_{0}\right) \cap M=\pi q_{m}\left(\beta_{1}\right) \cap M$. Denote this set by $S_{m}$.

Note that $y_{m}, t_{m}, S_{m} \in M$.

To see that $\beta_{0}, \beta_{1}$ satisfy $(*)$, fix $m \in N$. Let $\varphi(\alpha)$ be the conjunction of the following statements:

(a) $\alpha \in \bigcup \mathcal{G}_{m}$

(b) $y_{m} \subset q_{m}(\alpha)$;

(c) for every $\langle\xi, n\rangle \in S_{m} \times \omega,\langle\xi, n\rangle \in q_{m}(\alpha)$ iff $\langle\xi, n\rangle \in y_{m}$;

(d) for every $\xi \in S_{m} \cap H,\left\langle\xi, w_{\xi}(\alpha)\right\rangle \in q_{m}(\alpha)$ iff $\xi \in t_{m}$.

Note that all the parameters of $\varphi(\alpha)$ are from $M$, and that $\varphi\left(\beta_{0}\right)$ (as well as $\left.\varphi\left(\beta_{1}\right)\right)$ holds. Therefore, by standard reflection, $\varphi(\alpha)$ is true for infinitely many $\alpha \in M$, in fact,

$\psi$ : there is an infinite function $v$ such that $\operatorname{dom}(v) \subset c^{+}, \varphi(\alpha)$ and $v(\alpha)=q_{m}(\alpha)$ hold for every $\alpha \in \operatorname{dom}(v)$, and $\langle\pi v(\alpha)\rangle_{\alpha \in \operatorname{dom}(v)}$ forms an infinite $\Delta$-system with root $r_{v}=S_{m}$.

Since all parameters of $\psi$ are from $M$ we can choose a $v \in M$ as above. Let $\alpha \in A^{*}$ be such that (D) holds. We are going to show that $\left\{\beta_{0}, \beta_{1}\right\} \subset Q_{m}(\alpha)$. To see this, let $\xi_{0}<\xi_{1}<\cdots<\xi_{t-1}$ enumerate $\pi q_{m}(\alpha)$. By induction on $k=0, \ldots, t-1$ we are going to prove

$$
\text { if }\left\langle\xi_{k}, \cdot\right\rangle \in q_{m}(\alpha) \text {, then }\left\{\beta_{0}, \beta_{1}\right\} \subset Q_{\xi_{k}} .
$$

Let $0 \leq k \leq t-1$ and suppose that $\left(I_{j}\right)$ holds for $j<k$. In order to prove $\left(I_{k}\right)$, let $\left\langle\xi_{k}, \cdot\right\rangle \in q_{m}(\alpha)=v(\alpha)$.

We are going to split our argument into two cases and consider two subcases in each case.

Case 1. Suppose $\left\langle\xi_{k}, \cdot\right\rangle=\left\langle\xi_{k}, n\right\rangle$ for some $n \in \omega$.

Subcase 1(a). Suppose $\xi_{k} \in S_{m}$. Then, since (c) of $\varphi(\alpha)$ holds and $\left\langle\xi_{k}, n\right\rangle \in q_{m}(\alpha)$, it follows that $\left\langle\xi_{k}, n\right\rangle \in q_{m}\left(\beta_{i}\right)$ for $i=0,1$. Thus $\left\{\beta_{0}, \beta_{1}\right\} \subset Q_{\xi_{k} n}\left(=G_{\xi_{k} n}\right)$.

Subcase 1(b). Suppose $\xi_{k} \notin S_{m}=r_{v}$. Then, for $i=0,1$, it follows that $\alpha<\beta_{i}$ and $\left\langle Y_{\xi_{k}} \cap A^{*}, n\right\rangle \in u_{0}^{*}(\alpha)$ (i.e., $\left.\left\langle Y_{\xi_{k}} \cap A_{\beta i}, n\right\rangle \in u_{0 \beta_{i}}(\alpha)\right)$. By $\left(2_{\xi_{k}}\right)$ it follows that $g_{\xi_{k}}\left(\beta_{i}\right) \geq n$, i.e. $\beta_{i} \in G_{\xi_{k} n}=Q_{\xi_{k} n}$.

Case 2. Suppose $\left\langle\xi_{k}, \cdot\right\rangle=\left\langle\xi_{k}, \rho\right\rangle$ for some $\rho \in c^{+} \backslash \omega$. 
Subcase 2(a). Suppose $\xi_{k} \in S_{m}$. Then, since $\left(I_{j}\right)$ holds for $j<k$ and since $q_{m}(\alpha)$ satisfies $(\mathrm{F})$, it follows that

$$
\left\{\beta_{0}, \beta_{1}\right\} \subset T_{\xi_{k} m}(\alpha) \subset U_{\xi_{k} \rho}=U_{\xi_{k} w_{\xi_{k}}(\alpha)} .
$$

Furthermore, since $\xi_{k} \in S_{m} \cap H=r_{v} \cap H$, it follows that $U_{\xi_{k}} \cap\left(A^{*} \times A^{*}\right) \in u_{2}^{*}(\alpha)$ (i.e., $\left.U_{\xi_{k}} \cap\left(A_{\beta_{i}} \times A_{\beta_{i}}\right) \in u_{2 \beta_{i}}(\alpha)\right)$ for $i=0,1$. Thus by $\left(4_{\xi_{k}}\right.$-c) there are $\alpha_{0}, \alpha_{1} \in A^{*}$ such that $w_{\xi_{k}}\left(\beta_{i}\right)=w_{\xi_{k}}\left(\alpha_{i}\right)$ for $i=0,1$. Let us denote these common values by $\rho_{i}(i=$ $0,1)$. Since $\xi_{k} \in M$ and $\left\langle w_{\xi}\right\rangle_{\xi \in H} \in M, w_{\xi_{k}} \in M$. Since $\alpha_{i} \in M, \rho_{i}=w_{\xi_{k}}\left(\alpha_{i}\right) \in M$. Thus $\left\langle\xi_{k}, \rho_{i}\right\rangle \in M$ for $i=0,1$. Furthermore, since $\left\langle\xi_{k}, \rho\right\rangle=\left\langle\xi_{k}, w_{\xi_{k}}(\alpha)\right\rangle \in q_{m}(\alpha)$, by part (d) of $\varphi(\alpha), \xi_{k} \in t_{m}$, so $\left\langle\xi_{k}, \rho_{i}\right\rangle \in M \cap q_{m}\left(\beta_{i}\right)=y_{m}$ for $i=0,1$. By part (b) of $\varphi(\alpha), \mathrm{y}_{m} \subset q_{m}(\alpha)$, so $\left\langle\xi_{k}, \rho_{i}\right\rangle \in q_{m}(\alpha)$. Since $\left\langle\xi_{k}, \rho\right\rangle \in q_{m}(\alpha)$, this implies $\rho=\rho_{0}=\rho_{1}$; hence $\beta_{i} \in W_{\xi_{k} \rho_{i}}=W_{\xi_{k} \rho}$ for $i=0,1$.

Subcase 2(b). Suppose $\xi_{k} \notin S_{m}$. Then $\xi_{k} \in \pi q_{m}(\alpha) \cap H \backslash S_{m} \subset \pi v(\alpha) \backslash r_{v}$, so $U_{\xi_{k}} \cap\left(A^{*} \times A^{*}\right) \in u_{1}^{*}(\alpha)$, (i.e. $\left.U_{\xi_{k}} \cap\left(A_{\beta_{i}} \times A_{\beta_{i}}\right) \in u_{1 \beta_{i}}(\alpha)\right)$ for $i=0$, 1. Furthermore, $\alpha<\beta_{i}$, and since $\left(I_{j}\right)$ holds for $j<k$, and $q_{m}(\alpha)$ satisfies $(\mathrm{F})$, it follows that

$$
\left\{\beta_{0}, \beta_{1}\right\} \subset T_{\xi_{k} m}(\alpha) \subset U_{\xi_{k} \rho}=U_{\xi_{k} w_{\xi_{k}}(\alpha)} .
$$

Thus by $\left(4_{\xi_{k}}-\mathrm{b}\right), \omega_{\xi}\left(\beta_{i}\right)=w_{\xi_{k}}(\alpha)=\rho$ holds for $i=0,1$, i.e. $\left\{\beta_{0}, \beta_{1}\right\} \subset W_{\xi_{k}} \rho=$ $Q_{\xi_{k} \rho}$.

This concludes the proof of Theorem 1.1.

\section{Final Remarks, OPEN QUeStions}

A. As pointed out earlier, $Q X$ is not $\sigma$-discrete, because a $\sigma$-discrete space in which all points are $G_{\delta}$-sets has a quasi- $G_{\delta}$-diagonal. (Indeed, if $Y=\bigcup_{m \in \omega} Y_{m}$ is such a space (with each $Y_{m}$ a discrete subspace), then for each $y \in Y_{m}$, let us pick a sequence $\left\langle G_{y m k}\right\rangle_{k \in \omega}$ of open sets such that $\{y\}=\bigcap_{k \in \omega} G_{y m k}$ and $G_{y m k} \cap Y_{m}=\{y\}$ for every $k \in \omega$. Then $\left\langle\mathcal{G}_{m k}\right\rangle_{m, k \in \omega}$, where $\mathcal{G}_{m k}=\left\{G_{y m k}: y \in Y_{m}\right\}$, is a quasi-

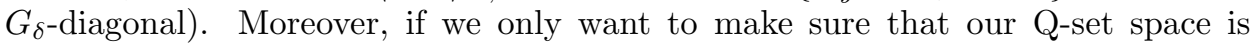
not $\sigma$-discrete, then the construction of $Q X$ can be done on $c$ instead of $c^{+}$, with minimal changes.

Theorem 2.1. There is a paracompact perfectly normal Q-set space of cardinality $c$.

It is interesting to note that all normal Q-set spaces of cardinality $\leq c$ have a $G_{\delta^{-}}$ diagonal (more generally, all cleavable spaces of cardinality $\leq c$ have a $G_{\delta}$-diagonal $[\mathrm{AS}]$ ); hence to get " $Q X$ has no (quasi-) $G_{\delta}$-diagonal" it was necessary to work on $c^{+}$instead of just $c$.

B. We can't hope that a Q-set space constructed in ZFC will have properties any closer to metrizability then being paracompact; indeed, under $V=L$, not only that there are no metrizable Q-set spaces, but there are no Q-set spaces with character $\leq c([\mathrm{R}],[\mathrm{H}],[\mathrm{BJ}])$.

Under $V=L$, every Q-set space is $\sigma$-left separated [BJ], so a non- $\sigma$-left-separated $\mathrm{Q}$-set space cannot be constructed in ZFC. Of course, under MA $+\neg \mathrm{CH}$, even the real line has Q-set subspaces (see Miller's paper in [KV], e.g.).

C. There are several natural questions which are left open.

Problem 1. Is there a connected normal Q-set space?

For Q-spaces, this is a question of A.V. Arhangel'skii [A2], who also points out that P. deCaux $[\mathrm{C}]$ constructed an infinite, regular, connected, $\sigma$-closed-discrete 
space. (Note that, of course, a $\sigma$-closed discrete space is a Q-space, but not a Q-set space.)

Problem 2. Is there a strong Q-set space in ZFC, i.e. a space $X$ such that all finite powers of $X$ are Q-set spaces? Can such a space be normal or paracompact?

(Note that under MA $+\neg \mathrm{CH}$, the real line has strong Q-set subspaces.)

Problem 3. Is there, in ZFC, a Q-set space of size $\omega_{1}$ ?

It is interesting to note that the answer is yes both under $\mathrm{CH}$ and $\operatorname{MA}\left(\omega_{1}\right)$. Under $\mathrm{CH}$ the space in $[\mathrm{B}]$ works, and the space of Theorem 2.1 is an example which is even paracompact. Under $\operatorname{MA}\left(\omega_{1}\right)$, any subset of cardinality $\omega_{1}$ of the real line is an example.

\section{REFERENCES}

[A1] A.V. Arhangel'skiǐ, The general concept of cleavability of a topological space, Top. Applications 44 (1992), 27-36. MR 93k:54047

[A2] A.V. Arhangel'skiř, Divisibility and cleavability of spaces, Recent Developments of General Topology and Applications, Math. Res., vol. 67, Akademie-Verlag, Berlin, 1992, pp. 13-26. MR 94e:54028

[AS] A.V. Arhangel'skiǐ and D. Shakhmatov, Pointwise approximation of arbitrary functions by countable families of continuous functions, Trudy Sem. Petrovsk 13 (1988), 206-227; English transl., J. Soviet Math. 50 (1990), 1497-1511. MR 89m:41023

[B] Z. Balogh, There is a Q-set space in ZFC, Proc. AMS 113 (1991), 557-561. MR 91m:54046

[BJ] Z. Balogh and H. Junnila, Totally analytic spaces under $V=L$, Proc. Amer. Math. Soc. 87 (1983), 519-527. MR 84j:54026

[C] P. deCaux, A nondegenerate $\sigma$-discrete Moore space which is connected, Colloq. Math. 41 (1979), 207-209. MR 81m:54058

[H] R.W. Hansell, Some consequences of $(V=L)$ in the theory of analytic sets, Proc. Amer. Math. Soc. 80 (1980), 311-319. MR 81j:54058

[J] H. Junnila, Some topological consequences of the Product Measure Extension Axiom, Fund. Math. 115 (1983), 1-8. MR 85e:54021

[KV] K. Kunen and J. Vaughan, eds., Handbook of Set-Theoretic Topology, North-Holland, 1984. MR 85k:54001

[R] G.M. Reed, On normality and countable paracompactness, Fund. Math 110 (1980), 145-152. MR 82d:54033

Department of Mathematics and Statistics, Miami University, Oxford, Ohio 45058

E-mail address: ZTBalogh@miavx1.muohio.edu 